\title{
Acute bronchospasm during passive exposure to bronchial provocation tests
}

\section{To the Editor:}

Bronchial challenge with direct stimuli, like histamine and methacholine, is very sensitive for diagnosing asthma and produces similar responses on a milligram-on-milligram or on a millimole-on-millimole basis [1-4]. American Thoracic Society (ATS)/European Respiratory Society (ERS) guidelines have proposed measures in order to protect technicians performing these tests, which include the use of good filters and ventilation, and have recommended that technicians with asthma should take extra precautions to minimise exposure or should avoid challenge testing. Performing methacholine/histamine challenge tests on technicians has also been thought to be useful [1]. Evidence however remains limited with only three published papers reporting some reaction to passive exposure in technicians [5-7], with two of these cited in the ATS/ERS guidelines [1]. One was a survey that only reported technicians' symptoms during challenge tests [5], another described two nurses who developed increased airway responsiveness after 2 years of regular practice of histamine and methacholine challenge tests, and the third paper reported episodic bronchospasm in a female technician known to have stable and well-treated asthma [7].

We have prospectively investigated the risk of acute bronchospasm during passive exposure to histamine. We invited symptomatic patients with newly diagnosed high levels of bronchial hyperreactivity (PD20 (provocative concentration causing a $20 \%$ fall in forced expiratory volume in 1 s) at first histamine dose) to agree to be passively exposed to histamine within 7 days of their first test in a manner comparable to the technician's passive exposure. We reasoned that if these patients showed no response to passive exposure, then the risk to asymptomatic technicians would be very small. The study was approved by the ethics committee (Saint-Pierre University Hospital, Brussels, Belgium) and participants filled in an informed consent form. For this exploratory and mainly descriptive study, a sample size of 12 patients was considered sufficient and the study was registered (NCT01937494).

13 adult patients with symptoms (i.e. dyspnoea, non-productive cough, chest tightness and wheezing) not diagnostic for asthma, and positive histamine provocation test at first dose were included. None of them received asthma treatment. During the diagnostic test, the patient was seated in the body box cabin whose door remained open. The technician remained close to the cabin (about $1 \mathrm{~m}$ ). In addition to the expiratory filter, a standard ventilation system was present in the room whose approximate surface area was $4 \mathrm{~m}^{2}$. During the passive exposure test, the patients remained close to the technician, while the subjects actively exposed were now volunteers with previously known negative results.

The conventional 2-min tidal breathing histamine challenge test protocol was followed using Jaeger APS Pro (ERICH Jaeger GmbH, Wurzburg, Germany) and DeVilbiss 646 (Sunrise Medical HHG Inc., Somerset, PA, USA) jet nebuliser with an output of $900 \mu \mathrm{L} \cdot \mathrm{min}^{-1}$ and a distribution range of $2.1 \mu \mathrm{m}[1,3]$. The expiratory filter was always changed after four challenge tests. We used a short protocol of five concentrations $(0.9 \%$ saline as initial baseline control, histamine 1 and/or $2 \mathrm{mg} \cdot \mathrm{mL}^{-1}, 4,8,16$ and $32 \mathrm{mg} \cdot \mathrm{L}^{-1}$ ) with the $1 \mathrm{mg} \cdot \mathrm{mL}^{-1}$ concentration being usually given to patients with the highest suspicion of severe bronchial hyperreactivity $[1,8]$. The forced expiratory volume in $1 \mathrm{~s}\left(\mathrm{FEV}_{1}\right)$ was obtained at each step about 30 and $90 \mathrm{~s}$ after nebulisation and the test was stopped if the FEV1 fall was equal to or exceeded $20 \%$ of baseline FEV1. The corresponding dose for a 20\% decrease in FEV1 (PD20) was then calculated and the results of active and passive exposure were compared.

13 patients (12 female) with normal FEV1 to forced vital capacity ratio (standardised residuals, $\mathrm{z}$-scores, between -1.630 and 0.260$)[9,10]$ and no contraindication to histamine challenge test [1] were included.

Symptoms at presentation included dyspnoea in 10 (77\%), cough in eight (62\%), chest tightness in two $(15 \%)$ and wheezing in two (15\%) patients. During active exposure to histamine, a positive reaction at the first dose was observed in all included patients with a PD20 $<60 \mu \mathrm{g}$ and a mean \pm SD FEV1 fall amounting to $830 \pm 270 \mathrm{~mL}$. During passive exposure, no reaction was observed in 12 (92\%) of the patients; one patient had a very mild reaction with a PD20 at $614 \mu \mathrm{g}$ (at a concentration of $16 \mathrm{mg} \cdot \mathrm{mL}^{-1}$ of histamine) (figure 1). None of the study patients felt chest tightness or showed cough during the passive exposure. No 


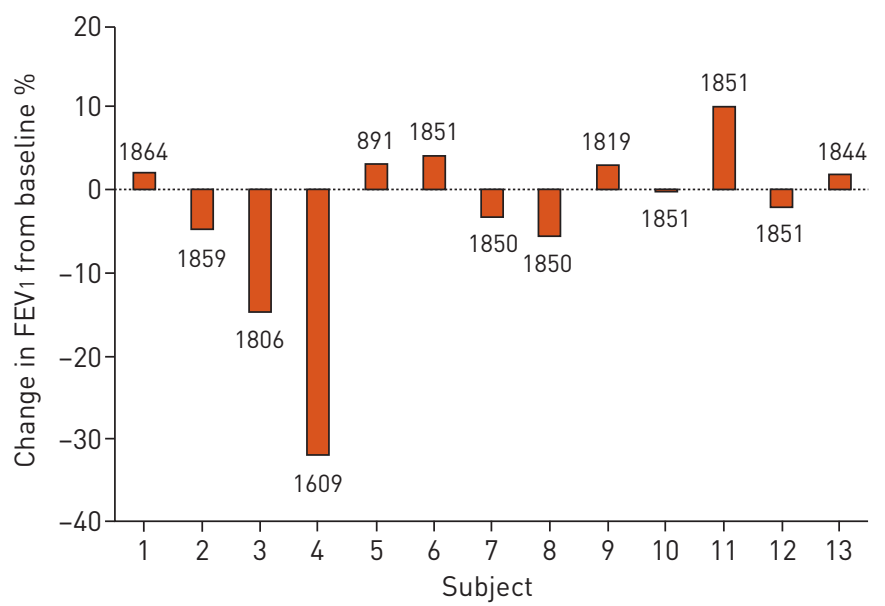

FIGURE 1 Results of the passive histamine challenge test with the subject's number on the $x$-axis and, on the $y$-axis, the subjects' percentage change in forced expiratory volume in $1 \mathrm{~s}$ (FEV 1 ) on completion of the test. The final dose of histamine (in $\mu \mathrm{g}$ ) is plotted for each subject. Only one subject (number 4) had a significant response with $>20 \%$ fall of $\mathrm{FEV}_{1}$ at a PD20 of $614 \mu \mathrm{g}(891 \mu \mathrm{g}$ total dose of histamine received). See text for further explanation.

relationship appeared between the $\mathrm{PD} 20$ of the first diagnostic test and the passive $\mathrm{PD} 20$ or the last used dose without reaction of the passive test.

These data show that symptomatic subjects with reaction to lowest doses during histamine challenge test show an absence of clinically significant response when they are exposed to histamine in conditions mimicking those of laboratory technicians performing the test. In contrast with previous studies that suggested a risk associated with passive exposure [5-7], mouthpiece and box valve on the expired side as well as adequate ventilation that minimised the exposure were used $[2,11]$. The study from LUNDGREN et al. [6] also suggested that repeated passive exposure may lead to airway hyperreactivity. This is however in contradiction with previous findings in asthmatic patients showing that histamine provocation tests can be repeated without the appearance of tachyphylaxis or increased sensitivity to histamine [12].

Finally, the absence of response to passive exposure in symptomatic highly hyperreactive patients suggests that there is no need for a previous challenge testing in asymptomatic technicians starting to work in a lung function department.

@ERSpublications

No need for bronchial challenge testing in asymptomatic technicians commencing work in a lung function department http://ow.ly/S2D3z

Maria Gabrovska, Emmanuel Lacombe, Marie Bruyneel and Vincent Ninane

Chest Service, Saint-Pierre University Hospital, Brussels, Belgium.

Correspondence: Maria Gabrovska, Chest Service, Saint-Pierre University Hospital, Rue Haute 322, 1000 Brussels, Belgium. E-mail: maria_gabrovska@stpierre-bru.be

Received: Jan 102015 | Accepted after revision: July 242015 | First published online: Sept 242015

Clinical trial: This study is registered at ClinicalTrials.gov with identifier number NCT01937494.

Conflict of interest: None declared.

\section{References}

1 Carpo RO, Casaburi R, Coates AL, et al. Guidelines for methacholine and exercise challenge testing - 1999. Am J Respir Crit Care Med 2000; 161: 309-329.

2 Cockroft DW. Bronchoprovocation methods: direct challenges. Clin Rev Allergy Immunol 2003; 24: 19-26.

3 Cockroft DW. Airway hyperresponsiveness in asthma: its measurement and clinical significance. Chest 2010; 138: Suppl. 2, S18-S24.

4 Higgins BG, Britton JR, et al. Comparison of histamine and methacholine for use in bronchial challenge tests in community studies. Thorax 1988; 43: 605-610.

5 Shapiro GG, Simon RA. Bronchoprovocation committee report. J Allergy Clin Immunol 1992; 89: 775-778.

6 Lundgren R, Söderberg M, Rosenhall L, et al. Development of increased airway responsiveness in two nurses performing methacholine and histamine challenge tests. Allergy 1992; 47: 188-189. 
7 Sen RP, Walsh TE. Accidental methacholine bronchoprovocation in a laboratory worker. Chest 1991; 99: 1053-1054.

8 Sterk PJ, Fabbri LM, Quanjer PH, et al. Airway Responsiveness: Standardised challenge testing with pharmacological, physical and sensitizing stimuli in adults. Eur Respir J 1993; 6: Suppl. 16, 53-83.

9 Quanjer PH, Stanojevic S, Cole TJ, et al. Multi-ethnic reference values for spirometry for the 3-95 year age range: the global lung function 2012 equations. Eur Respir J 2012; 40: 1324-1343.

10 Miller MR. Does the use of per cent of predicted have any evidence base? Eur Respir J 2015; 45: 322-323.

11 Juniper EF, Syty-Golda M, Harggreave FE. Histamine inhalation tests: inhalation of aerosol via a facemask versus a valve box with mouthpiece. Thorax 1984; 39: 556-557.

12 Ruffin RE, Alpers JH, Cockett AJ, et al. Repeated histamine inhalation tests in asthmatic patients. J Allergy Clin Immunol 1981; 67: 285-289.

\title{
Nasal potential difference of carriers of the W493R ENaC variant with non-cystic fibrosis bronchiectasis
}

\author{
To the Editor:
}

Common underlying conditions leading to bronchiectasis are infection, immune deficiency or cystic fibrosis (CF) [1]. Transgenic mice that overexpress the amiloride-sensitive epithelial sodium channel ENaC mimic the typical features of CF lung disease [2], and correspondingly, it has been proposed that ENaC hyperactivity may predispose to bronchiectasis [3]. The ENaC ion channel consists of three subunits which are encoded by the genes SCNN1A, SCNN1B and SCNN1G. Sequencing of these three genes in 71 subjects with CF-like disease in whom a mutation could not be identified on both CFTR (cystic fibrosis transmembrane conductance regulator) genes uncovered singular cases of disease caused by mutations in SCNN1A [3] and $S C N N B$ [4], and a higher incidence of $E N a C$ polymorphisms [3]. Of nine identified amino acid sequence polymorphisms, one variant in the alpha subunit, named p.W493R-SCNN1A, encoded a hyperactive ENaC channel when tested in a heterologous expression system [3]. Hence AzAD et al. [3] proposed that the p.W493R-SCNN1A ENaC variant could be a global risk factor for the development of lung diseases.

We tested this hypothesis by screening 69 adults with non-CF bronchiectasis from the adult bronchiectasis clinic at Hannover Medical School for the carriage of p.W493R-SCNN1A (rs5742912) (table 1). The study was conducted with the approval of the Ethics Committee of Hannover Medical School (study number: 5391). CF had been excluded by chloride concentrations below the pathological range in the sweat test and/or the absence of any disease-causing mutation in the CFTR coding region in genetic testing. Genomic DNA was amplified with the forward primer 5'-TGCAGGTTAGTGTCCCCTTC and the reverse mismatch primer 5'-CTGGGATGTCACCGATGG by PCR. The 166-bp long PCR product was digested with BseJI (Fermentas; Thermo Fisher Scientific, Vilnius, Lithuania), which cleaves the common W493 allele, and separated by $4 \%$ agarose gel electrophoresis. Six of the 69 subjects with non-CF bronchiectasis were identified as heterozygous carriers of p.W493R-SCNN1A. Thus the incidence of p.W493R-SCNN1A of $8.7 \%$ was significantly higher in the investigated disease cohort than that of $3.1 \%$ in a previously studied healthy control cohort of 739 Mid-European subjects of similar ethnic descent [3] $(p=0.022$, Fisher's exact test). This data demonstrates an association between the carrier status for p.W493R and the manifestation of bronchiectasis.

Next, we wanted to know whether carriage of p.W493R-SCNN1A translates into higher sodium channel activity as indicated by a larger hyperpolarisation response to amiloride in nasal potential difference measurements (NPD) of the upper respiratory epithelium. Five of the six p.W493R carriers were available for an assessment by NPD. NPD measurements were performed according to the Standard Operating Procedure NPD_EU001, version 1.7 (March 2013) of the European Cystic Fibrosis Society Diagnostic Network Working Group, which primarily differs from a publicly available protocol [5] by superfusion of room temperature solutions through a Marquat catheter (model I0202US; Marquat Genie Biomedical, Boissy-Saint-Leger, France). Two subjects presented normal NPD tracings, and two subjects showed a normal response to amiloride, but only a minute response upon exposure to chloride-free solution consistent with the diagnosis of a CFTR-related disorder [6, 7]. However, one subject, a 58-year old female 